\title{
Suitability of the unidirectional approach for describing laser-driven terahertz emission
}

\author{
$\underline{\text { J. Déchard }^{1}}$, P. González de Alaiza Martínez ${ }^{2}$, A. Nguyen ${ }^{1}$, I. Thiele ${ }^{2}$, S. Skupin ${ }^{2}$, and L. Bergé ${ }^{1}$ \\ 1. CEA-DAM, DIF, 91297 Arpajon, France \\ 2. Univ. Bordeaux - CNRS - CEA, Centre Lasers Intenses et Applications, UMR 5107, 33405 Talence, France
}

Over the past decade, the increasing potential of terahertz $(\mathrm{THz})$ radiation has stimulated intensive efforts to develop efficient emitters based on the ionization of gases by ultrashort laser pulses [1]. In order to properly calibrate dedicated experiments, nonlinear propagation codes have to be particularly accurate to describe the lowfrequency part of the pulse spectrum. Usually, solving full Maxwell models accounting for multiple ionization processes as well as optical and plasma nonlinear effects is computationally expensive when simulating long propagation ranges. Therefore, reduced models such as the Unidirectional Pulse Propagation Equation (UPPE) [2] are often preferred, assuming that the forward propagating component conveys the major part of the laser energy. So far, no detailed numerical comparison has been performed to confirm the validity of this UPPE approach, which is widely used in the scope of laser-driven $\mathrm{THz}$ generation [3]. Here, we examine by means of 1D simulations the differences occurring in the $\mathrm{THz}$ pulse spectra and fields when these quantities are described either by a full Maxwell-fluid (MAXFLU) model, encompassing both backward and forward propagations, or by its UPPE approximation, modeling only the forward propagating wave.

Figure 1(a) shows the spectra of the forward, transverse $\mathrm{THz}$ fields parallel to the laser polarization axis and produced in argon by a 50-fs two-color pulse with $1 \mu \mathrm{m}$ fundamental pump at increasing propagation distances, when using UPPE (red curves) and the MAXFLU model (blue curves). When the backward-propagation operator is dropped out, the fundamental linear modes beating at the electron plasma frequency $\omega_{\mathrm{pe}}$ are lost and no plasma opacity is allowed, which results in the development of oscillatory components in the UPPE spectrum in the frequency range $v<\omega_{\mathrm{pe}} / 2 \pi$. In contrast, the MAXFLU spectrum is dominated by plasma current oscillations, which prevail as long as the propagation distances remain of a few plasma skin depths $\left[c / \omega_{\mathrm{pe}} \approx 3 \mu \mathrm{m}\right.$ for Figure 1(a)]. Over longer distances, however, both UPPE and MAXFLU spectra merge, as photocurrents become the dominating source in the $\mathrm{THz}$ generation process. This property is refound for a more intense, single-color pulse with few-cycle durations [Figure 1(b), $c / \omega_{\mathrm{pe}} \approx 1 \mu \mathrm{m}$ ], for which we expect a dominant role of the photocurrents [4].
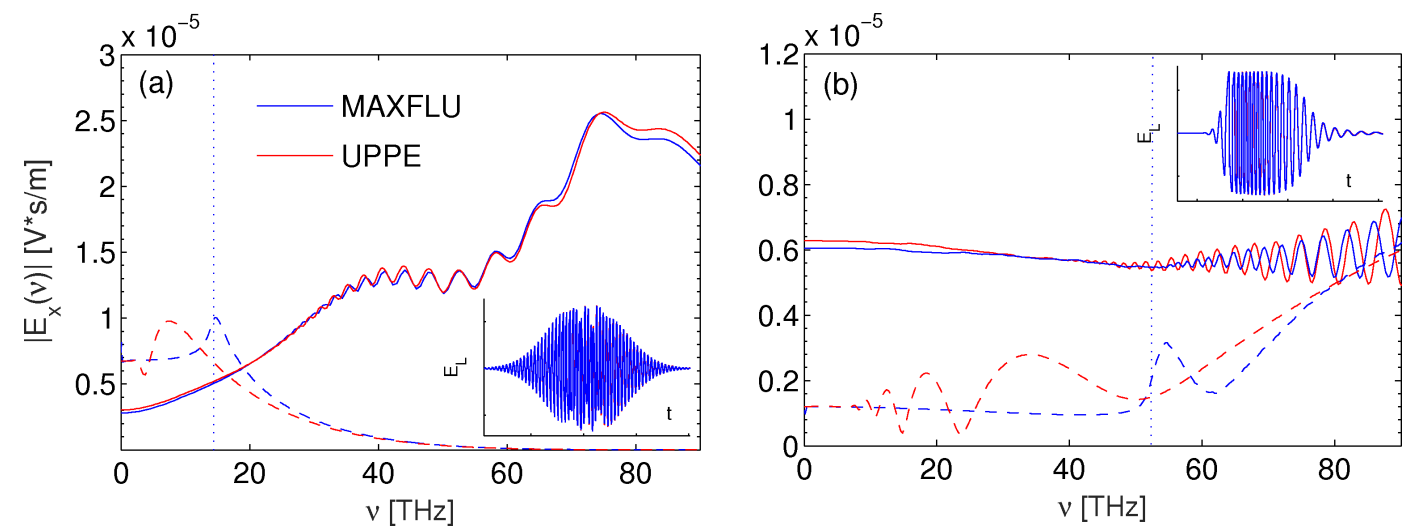

Figure 1 Spectra of the THz transverse fields at different propagation distances in argon computed from the Maxwell-fluid code MAXFLU (blue curves) and the UPPE code (red curves) for (a) a 50-fs two-color pulse with $150 \mathrm{TW} / \mathrm{cm}^{2}$ intensity at $z=10 \mu \mathrm{m}$ (dashed curves) and $z=1 \mathrm{~cm}$ (solid curves); (b) a 8 -fs single-color pulse of $10^{15} \mathrm{~W} / \mathrm{cm}^{2}$ intensity at $z=8 \mu \mathrm{m}$ (dashed curves) and $z=5 \mathrm{~mm}$ (solid curves). The vertical dotted curves indicate the electron plasma frequency $v_{p e}=\omega_{\mathrm{pe}} / 2 \pi$. Insets illustrate the laser field profiles in time at their respective larger distances of propagation.

In summary, we demonstrate that in a one-dimensional geometry the UPPE model that only governs the forward pulse component is able to provide similar spectra to a bidirectional Maxwell-fluid model over distances from which photocurrents mainly drive $\mathrm{THz}$ pulse generation.

\section{References}

[1] K. Y. Kim, A. J. Taylor, J. H. Glownia, and G. Rodriguez,"Coherent control of terahertz supercontinuum generation in ultrafast laser-gas interactions," Nat. Photon. 2, 605 (2008).

[2] M. Kolesik, J. V. Moloney, and M. Mlejnek, “Unidirectional optical pulse propagation equation,” Phys. Rev. Lett. 89, 283902 (2002).

[3] V. A. Andreeva et al., "Ultrabroad terahertz spectrum generation from an air-based filament plasma," Phys. Rev. Lett. 116, 063902 (2016).

[4] I. Thiele et al. "Theory of terahertz emission from femtosecond-laser-induced microplasmas," Phys. Rev. E 94, 063202 (2016). 